\title{
A numerical characteristic of extreme values*
}

\section{TAKAAKI SHIMURA}

A numerical characteristic of large random numbers is studied. Let $F$ be a distribution on the real numbers with infinite endpoint. $X$ denotes a random variable with distribution $F$. Consider the transformation for a decimal number $d_{1} d_{2} d_{3} \ldots d_{n} . d_{n+1} \ldots$ in $\left[10^{n-1}, 10^{n}\right)$ to $0 . d_{2} d_{3} \ldots$ in $[0,1)$. We are interested in the distribution of transformed $X$ for large $X$, which implies the behavior of the large random number except the first figure. It is shown that the distribution of transformed $X$ conditioned by the first figure converges as $X$ becomes large for most distributions. Moreover, it turns out that the limit distribution depends on the tail behavior of $F$ and the first figure. A similar problem for distributions with finite endpoints is also considered. In this case, the distance until the endpoint is a matter of concern and parallel results to the ones for infinite endpoint case are given.

AMS 2000 SUbJeCt Classifications: Primary 60E05, $60 \mathrm{~F} 09$.

KEYWORDS AND PHRASES: Random number, Regular variation, П-variation.

\section{INTRODUCTION}

We investigate a numerical characteristic for large random numbers. For a positive random number $X=$ $d_{1} d_{2} d_{3} \ldots d_{n} \cdot d_{n+1} \ldots$, we call $d_{m}$ the $m$ th figure of $X$. Our interest is in the distribution of these figures. We assume $F$, the distribution of $X$, has an infinite endpoint: $\sup \{x: F(x)<1\}=\infty$. Define a normalized random variable $Y$ on $[0,1)$ as

$$
Y=X / 10^{N-1}-K,
$$

where $N$ is the number of figures before the decimal point when it is expressed in the decimal system and $K$ is the first figure of $X: 10^{N-1} \leq X<10^{N}$ and $X / 10^{N-1}-1<K \leq$ $X / 10^{N-1}$. $Y$ expresses the behavior of $X$ except the first figure. We consider the conditional distribution

$$
F^{k, n}(y)=P(Y \leq y \mid K=k, N=n),
$$

for $k=1,2, \ldots, 9$. Our main interest is in the behavior of $F^{k, n}$ as $n \rightarrow \infty$ for each $k$.

* This study was carried out under the ISM Cooperative Research Program (2012-ISM · CRP-5009, 2013-ISM · CRP-5012).
In preparation, we introduce the regular variation and related derivations to characterize the tail behaviors (see [1], [3]). A measurable positive function $f(x)$ on $[0, \infty)$ is said to be regularly varying with index $\rho(\in \mathbf{R})$ if $\lim _{x \rightarrow \infty} f(\lambda x) / f(x)=\lambda^{\rho}$ for each $\lambda>0$. The case of $\rho=0$ is called slowly varying. If $\lim _{x \rightarrow \infty} f(\lambda x) / f(x)=\infty$ for each $\lambda>1$ (resp. $\lambda<1$ ), it is called to be rapidly varying with index $\infty$ (resp. $-\infty$ ). A measurable positive non-decreasing function $f(x)$ is said to be $\Pi$-varying if there exist $a(x)>0$ and $b(x)$ such that

$$
\lim _{x \rightarrow \infty}(f(\lambda x)-b(x)) / a(x)=\log \lambda \quad \text { for each } \lambda>0 .
$$

We express the sets of regularly or rapidly varying functions with index $\rho \in[-\infty, \infty]$ and $\Pi$-varying functions as $\mathbf{R V}_{\rho}$ and $\Pi$, respectively. It is known that $\Pi \subset \mathbf{R V}_{\mathbf{0}}$.

Denote the tail of $F$ by $\bar{F}(x)=1-F(x)$. We say that $F$ has a regularly (resp. rapidly) varying tail if $\bar{F}(x)$ is regularly (resp. rapidly) varying. In the same way, we say that $F$ has a $1 / \Pi$-varying tail if $1 / \bar{F}(x) \in \Pi$ for convenience. Most well-known distributions have one of these tails, rapidly varying, regularly varying and $1 / \Pi$-varying. The normal, the exponential, the Poisson and the log-normal distribution have a rapidly varying tail. Thus wide variety of distributions are included in this class. The Pareto distribution, the stable distribution like the Cauchy distribution and the F-distribution have a regularly varying tail. Although distributions with $1 / \Pi$ tail do not seem to be so familiar, the log-Cauchy distribution and the distribution with $F(x)=$ $1-(\log x)^{-1}(x \geq e)$, both have a $1 / \Pi$ tail. [2] calls slowly varying tail super-heavy tail and studies a test to distinguish between heavy and super-heavy tailed distributions.

Our main result is that the limit distribution of $F^{k, n}$ exists for these three types of tails. In the case of a rapidly varying tail, the limit distribution is the delta distribution at 0 (The distribution concentrated at a single point 0 ) for every $k$. In the case of regularly varying tails, a nondegenerate limit distribution appears and it depends on $k$. The limit distribution for $1 / \Pi$-varying tail is identical with the limit of the limit distributions of the regularly varying case.

Some properties of the non-degenerate limit distributions are studied. For the degenerate case, the rate of convergence to the delta distribution is shown.

A similar problem for distributions with finite endpoints is also considered. In this case, the distance until the endpoint is a matter of concern and parallel results to the ones for infinite endpoint case are given. 
The limit distribution of $F^{k, n}$ is shown in Section 2. In Section 3 , the property of limit distribution is investigated.

\section{LIMIT DISTRIBUTION FOR EXTREME VALUES}

\subsection{Limit distribution for large random numbers}

We consider the limit distribution of the normalized random large number as $n$ goes to infinity. $F^{k, n}(y)=P(Y \leq$ $y \mid K=k, N=n)$ is written as

$$
F^{k, n}(y)=\left\{\begin{array}{l}
0 \quad \text { for } y<0 \\
F\left[k 10^{n-1},(k+y) 10^{n-1}\right] \\
/ F\left[k 10^{n-1},(k+1) 10^{n-1}\right) \\
\quad \text { for } 0 \leq y<1 \\
1 \quad \text { for } y \geq 1
\end{array}\right.
$$

The following is our main result. $\lim _{n \rightarrow \infty} F^{k, n}(y)$ exists for most distributions and the limit distributions are restricted to three types depending on the tail behavior of $F$ and the first figure $k$.

Theorem 2.1. (i) If $\bar{F}(x) \in \mathbf{R V}_{-\infty}$, then for every $k=$ $1,2, \ldots, 9$,

$$
\lim _{n \rightarrow \infty} F^{k, n}(y)=1_{\{y \geq 0\}}
$$

where $1_{A}$ is the indicator function for a set $A$.

(ii) If $\bar{F}(x) \in \mathbf{R V}_{-\alpha}(\alpha>\mathbf{0})$, then for $0 \leq y \leq 1$,

$$
\lim _{n \rightarrow \infty} F^{k, n}(y)=\frac{1-\left(1+\frac{y}{k}\right)^{-\alpha}}{1-\left(1+\frac{1}{k}\right)^{-\alpha}} .
$$

(iii) If $1 / \bar{F}(x) \in \Pi$, then for $0 \leq y \leq 1$,

$$
\lim _{n \rightarrow \infty} F^{k, n}(y)=\frac{\log \left(1+\frac{y}{k}\right)}{\log \left(1+\frac{1}{k}\right)} .
$$

Proof.

$$
\begin{aligned}
F^{k, n}(y) & =P(Y \leq y \mid K=k, N=n) \\
& =\frac{P\left(k 10^{n-1} \leq X \leq(k+y) 10^{n-1}\right)}{P\left(k 10^{n-1} \leq X<(k+1) 10^{n-1}\right)} .
\end{aligned}
$$

(i)

$$
F^{k, n}(y)=\frac{1}{1+I}
$$

where

$$
I=\frac{P\left((k+y) 10^{n-1}<X<(k+1) 10^{n-1}\right)}{P\left(k 10^{n-1} \leq X \leq(k+y) 10^{n-1}\right)} .
$$

376 T. Shimura

$$
\begin{aligned}
I & \leq \frac{\bar{F}\left((k+y) 10^{n-1}\right)}{P\left(\left(k+\frac{1}{2} y\right) 10^{n-1}<X \leq(k+y) 10^{n-1}\right)} \\
& \sim \frac{\bar{F}\left((k+y) 10^{n-1}\right)}{\bar{F}\left(\left(k+\frac{1}{2} y\right) 10^{n-1}\right)} \rightarrow 0 \quad(n \rightarrow \infty)
\end{aligned}
$$

for $y>0(\sim$ denotes that the ratio of both sides goes to 1$)$, we have (1). In the cases of (ii) and (iii), the last term of (4) is asymptotically equal to

$$
\begin{aligned}
& \frac{\bar{F}\left(k 10^{n-1}\right)-\bar{F}\left((k+y) 10^{n-1}\right)}{\bar{F}\left(k 10^{n-1}\right)-\bar{F}\left((k+1) 10^{n-1}\right)} \\
= & \frac{1-\bar{F}\left((k+y) 10^{n-1}\right) / \bar{F}\left(k 10^{n-1}\right)}{1-\bar{F}\left((k+1) 10^{n-1}\right) / \bar{F}\left(k 10^{n-1}\right)} .
\end{aligned}
$$

If $\bar{F}(x) \in \mathbf{R V}_{-\alpha}(\alpha>0)$, then $\lim _{n \rightarrow \infty} \bar{F}\left((k+y) 10^{n-1}\right) /$ $\bar{F}\left(k 10^{n-1}\right)=(1+y / k)^{-\alpha}$. If $1 / \bar{F}(x) \in \Pi$, then $\bar{F}\left(k 10^{n-1}\right)-$ $\bar{F}\left((k+y) 10^{n-1}\right) \sim \log \left(1+\frac{y}{k}\right) a\left(k 10^{n-1}\right) \bar{F}\left(k 10^{n-1}\right) \bar{F}((k+$ y) $\left.10^{n-1}\right) \sim \log \left(1+\frac{y}{k}\right) a\left(k 10^{n-1}\right)\left(\bar{F}\left(k 10^{n-1}\right)\right)^{2}$, where in the last asymptotical equivalence we use the fact that $\bar{F}(x) \in$ $\mathbf{R V}_{0}$. Thus we get (2) and (3).

Let

$$
G_{\alpha}^{k}(y)=\frac{1-\left(1+\frac{y}{k}\right)^{-\alpha}}{1-\left(1+\frac{1}{k}\right)^{-\alpha}} \text { and } G_{0}^{k}(y)=\frac{\log \left(1+\frac{y}{k}\right)}{\log \left(1+\frac{1}{k}\right)} .
$$

Remark 2.1. (a) (i) and (iii) are regarded as the limit of (ii): $G_{\alpha}^{k}(y)$ converges to $\delta_{0}$ and $G_{0}^{k}$ as $\alpha \rightarrow \infty$ and $\alpha \rightarrow$ 0 , respectively.

(b) If $F$ is the Pareto distribution, $F^{k, n}(y)=G_{\alpha}^{k}(y)$ holds for $k 10^{n-1} \geq \inf \{x: F(x)>0\}$.

Although the assumption of (iii) looks slightly strict, we can not relax the condition to slowly varying tails as we show below.

Theorem 2.2. For arbitrary distribution $F$ with slowly varying tail and arbitrary distribution $G$ on $[0,1)$, there exists a distribution $F_{G}$ such that $\lim _{x \rightarrow \infty} \bar{F}_{G}(x) / \bar{F}(x)=1$ and $F_{G}^{k, n}=G$.

Proof. Let $X, N$ and $K$ be the same as before. Put $X_{1}=$ $K 10^{N-1}$ and $X_{2}=X-X_{1}$. Since $X_{1} \leq X<2 X_{1}$, we have $P(X>x) \sim P\left(X_{1}>x\right)$. Let $Z$ be a random variable with distribution $G$ and set $X_{3}=X_{1}+10^{N-1} Z$. Then $P(X>$ $x) \sim P\left(X_{1}>x\right) \sim P\left(X_{3}>x\right)$. The distribution of $X_{3}$ is a desired one as $F_{G}$.

The following shows the rate of converge to $\delta_{0}$ in (i) of Theorem 2.1.

Theorem 2.3. Assume that $\bar{F}(x) \in \mathbf{R V}_{-\infty}, F$ is absolutely continuous and its hazard function $h(t)$ belongs to $\mathbf{R V}_{\rho}(\rho \geq$ $-\mathbf{1})$. For $0 \leq y<1$,

$$
\lim _{n \rightarrow \infty} \frac{1}{10^{n-1} h\left(10^{n-1}\right)} \log \overline{F^{k, n}}(y)=-c(\rho, k, y),
$$


where

$c(\rho, k, y)= \begin{cases}(\rho+1)^{-1}\left\{(k+y)^{\rho+1}-k^{\rho+1}\right\} & \rho>-1 \\ \log \left(1+\frac{y}{k}\right) & \rho=-1\end{cases}$

Proof. We can express $\bar{F}(x)$ as

$$
\bar{F}(x)=\bar{F}(a) \exp \left\{-\int_{a}^{x} h(t) d t\right\},
$$

where $a$ is a constant. By $\bar{F}(x) \in \mathbf{R V}_{-\infty}$,

$$
\begin{aligned}
\overline{F^{k, n}}(y) & =\frac{\bar{F}\left((k+y) 10^{n-1}\right)-\bar{F}\left((k+1) 10^{n-1}\right)}{\bar{F}\left(k 10^{n-1}\right)-\bar{F}\left((k+1) 10^{n-1}\right)} \\
& \sim \frac{\bar{F}\left((k+y) 10^{n-1}\right)}{\bar{F}\left(k 10^{n-1}\right)} .
\end{aligned}
$$

Thus

$$
\begin{aligned}
\log \overline{F^{k, n}}(y) & =\log \frac{\bar{F}\left((k+y) 10^{n-1}\right)}{\bar{F}\left(k 10^{n-1}\right)}+o(1) \\
& =-10^{n-1} \int_{k}^{k+y} h\left(10^{n-1} t\right) d t+o(1) . \\
& \frac{1}{10^{n-1} h\left(10^{n-1}\right)} \log \overline{F^{k, n}}(y) \\
= & -\frac{1}{h\left(10^{n-1}\right)} \int_{k}^{k+y} h\left(10^{n-1} t\right) d t+\frac{o(1)}{10^{n-1} h\left(10^{n-1}\right)} .
\end{aligned}
$$

The first term of the right-hand side goes to $-\int_{k}^{k+x} t^{\rho} d t=-c(\rho, k, y)$ as $n \rightarrow \infty$. While the second term goes to 0 because $\lim _{t \rightarrow \infty} t h(t)=\infty$ from the rapid variation of $\bar{F}$.

Theorem 2.3 shows the relation between the convergence rate and the first figure $k . c(\rho, k, y)$ implies the speed of convergence. $c(\rho, k, y)$ is a decreasing function of $k$ for $-1 \leq$ $\rho<0$. In the case of $\rho=0, F$ has a slowly varying hazard function and $c(0, k, y)$ does not depend on $k$. Especially, $F^{k, n}$ does not depend on $k$ if $F$ is an exponential distribution. $c(\rho, k, y)$ is an increasing function of $k$ for $\rho>0$.

\subsection{Limit distribution for small random numbers}

From now on, we deal with distributions with finite endpoint and consider the length until the endpoint. We assume $F$, the distribution of $X$, has a finite endpoint. For simplicity, let the endpoint be $0: \sup \{x: F(x)<1\}=0$. Define a normalized random variable $Y$ on $[0,1)$ as

$$
Y=-10^{N} X-K,
$$

where $K$ is the first non-zero figure of $X$ and $N$ is the number of zeros before the first non-zero figure: $-10^{-N+1}<X \leq$ $-10^{-N}$ and $-10^{N} X-1<K \leq-10^{N} X . Y$ expresses the behavior of $X$ except the first non-zero figure. The following conditional distribution is considered.

$$
F^{k, n}(y)=P(Y \leq y \mid K=k, N=n),
$$

for $k=1,2, \ldots, 9$. As the large case, the behavior of $F^{k, n}$ as $n \rightarrow \infty$ for each $k$ is investigated. We show the results without proofs since they are similar to the ones for the large case.

Theorem 2.4. (i) If $\bar{F}(-1 / x) \in \mathbf{R V}_{-\infty}$, then for every $k=1,2, \ldots, 9$,

$$
\lim _{n \rightarrow \infty} F^{k, n}(y)=1_{\{y \geq 1\}},
$$

where $1_{A}$ is the indicate function of a set $A$.

(ii) If $\bar{F}(-1 / x) \in \mathbf{R V}_{\alpha}(\alpha<\mathbf{0})$, then for $0 \leq y \leq 1$,

$$
\lim _{n \rightarrow \infty} F^{k, n}(y)=\frac{\left(1+\frac{y}{k}\right)^{-\alpha}-1}{\left(1+\frac{1}{k}\right)^{-\alpha}-1} .
$$

(iii) If $1 / \bar{F}(-1 / x) \in \Pi$, then for $0 \leq y \leq 1$,

$$
\lim _{n \rightarrow \infty} F^{k, n}(y)=\frac{\log \left(1+\frac{y}{k}\right)}{\log \left(1+\frac{1}{k}\right)} .
$$

Example 2.1. (a) The exponential distribution and the log-normal distribution have a rapidly varying tail at their endpoint.

(b) The Beta distribution and the Pareto distribution have a regularly varying tail at their endpoint.

The limit distributions are identical with an extension of $G_{\alpha}^{k}(\alpha \geq 0)$ in Theorem 2.1 to non-positive parameters $\alpha$ $(\leq 0)$. We use the same notation for these limit distributions. However, the case of $\alpha \leq 0$ is more various than the case of $\alpha \geq 0$. The densities of the latter are always decreasing. On the other hand, the densities of the former have three patterns: decreasing $(-1<\alpha \leq 0)$, constant $(\alpha=-1)$ and increasing $\alpha<-1$. This two parameter distribution class is investigated in the following section in detail.

Theorem 2.5. For arbitrary distribution $F$ with slowly varying tail at 0 and arbitrary distribution $G$ on $[0,1)$, there exists a distribution $F_{G}$ such that $\lim _{x \uparrow 0} \bar{F}_{G}(x) / \bar{F}(x)=1$ and $F_{G}^{k, n}=G$.

Theorem 2.6. Assume that $\bar{F}(-1 / x) \in \mathbf{R V}_{-\infty}$ is absolutely continuous and its hazard function satisfies $h(-1 / t) \in$ $\mathbf{R V}_{\rho}(\rho \geq \mathbf{1})$. For $0<y \leq 1$

$$
\lim _{n \rightarrow \infty} \frac{10^{n}}{h\left(-10^{-n}\right)} \log F^{k, n}(y)=-\tilde{c}(\rho, k, y),
$$

where

$\tilde{c}(\rho, k, y)= \begin{cases}(\rho-1)^{-1}\left\{(k+y)^{1-\rho}-(k+1)^{1-\rho}\right\} & \rho>1 \\ \log \left(\frac{k+1}{k+y}\right) & \rho=1\end{cases}$ 


\section{PROPERTY OF LIMIT DISTRIBUTIONS}

\subsection{Dependency on the tail index and the first figure}

In this subsection, we study the limit distribution $G_{\alpha}^{k}$ $(\alpha \in(-\infty, \infty), k=1,2, \ldots, 9)$. We consider how $G_{\alpha}^{k}$ varies depending on $\alpha$ and $k$. It is shown that the density function and the distribution function have monotonicity in various senses. The density functions of $G_{\alpha}^{k}(\alpha \in(-\infty, \infty))$ denoted by $p_{\alpha}^{k}(y)$ are given as

$p_{\alpha}^{k}(y)=\frac{\alpha k^{-1}\left(1+\frac{y}{k}\right)^{-\alpha-1}}{1-\left(1+\frac{1}{k}\right)^{-\alpha}} \quad$ and $\quad p_{0}^{k}(y)=\frac{1}{\log \left(1+\frac{1}{k}\right)} \frac{1}{k+y}$

for $0 \leq y \leq 1$.

Proposition 3.1. (i) For each $k, p_{\alpha}^{k}(y)$ is a decreasing (resp. constant, increasing) function of $y$ and $\alpha>-1$ (resp. $\alpha=-1, \alpha<-1$ ).

(ii) $p_{\alpha}^{k}(0)$ is an increasing function of $\alpha$ for each $k$. While $p_{\alpha}^{k}(1)$ is decreasing function of $\alpha$ for each $k$.

(iii) $p_{\alpha}^{k}(0)$ is a decreasing (resp. constant, increasing) function of $k$ for $\alpha>-1$ (resp. $\alpha=-1, \alpha<-1$ ).

$p_{\alpha}^{k}(1)$ is an increasing (resp. constant, decreasing) function of $k$ for $\alpha>-1$ (resp. $\alpha=-1, \alpha<-1)$.

Proof. (i) is obvious. Direct calculations lead (ii) and (iii).

Proposition 3.2. (i) $G_{\alpha}^{k}(y)$ is an increasing function of $\alpha$ for each $k$ and $y$.

(ii) $G_{\alpha}^{k}(y)$ is a decreasing (resp. constant, increasing) function of $k$ for $\alpha>-1$ (resp. $\alpha=-1, \alpha<-1$ ).

Proposition 3.2 is proved by direct calculation. It is slightly troublesome and omitted.

Denote the mean of the limit distributions $G_{\alpha}^{k}$ by $M_{\alpha}^{k}$. Noticing $M_{\alpha}^{k}=1-\int_{0}^{1} G_{\alpha}^{k}(y) d y$, we reach a corollary.

Corollary 3.1. (i) $M_{\alpha}^{k}$ is a decreasing function of $\alpha$ for each $k$.

(ii) $M_{\alpha}^{k}$ is an increasing (resp. constant, decreasing) function of $k$ for $\alpha>-1$ (resp. $\alpha=-1, \alpha<-1$ ).

\subsection{Limit distribution of $\boldsymbol{m}$ th figure}

We take an interest in the distribution of $m$ th figure of an extreme value. Instead, we study the distribution of $m$ th figure of a random variable possessing the distribution function $G_{\alpha}^{k}$. Note that here the $m$ th figure is defined as in the beginning of Section 1. Denote the distribution by $H_{m}^{k}(k=1,2, \ldots, 9, m=2,3, \ldots) . H_{m}^{k}$ is a distribution on $\{0,1, \ldots, 9\}$ and $H_{m}^{k}(\{j\})=H_{m}^{k}(j)$ is written as

$$
\begin{array}{r}
H_{m}^{k}(j)=\sum_{l=0}^{10^{m-2}-1} G_{\alpha}^{k}\left[\frac{l}{10^{m-2}}+\frac{j}{10^{m-1}}, \frac{l}{10^{m-2}}+\frac{j+1}{10^{m-1}}\right) \\
\text { for } j=0,1, \ldots, 9 .
\end{array}
$$

Although $H_{m}^{k}(j)$ is monotone for $j$ from Proposition 3.1, this property disappears as $m$ goes to $\infty$ as follows.

Theorem 3.1. For each $k, H_{m}^{k}$ converges to the uniform distribution on $\{0,1, \ldots, 9\}$ as $m \rightarrow \infty$.

Proof. From the monotonicity, it is enough to show that $\lim _{m \rightarrow \infty} H_{m}^{k}(0)=10^{-1}$.

$$
\begin{aligned}
H_{m}^{k}(0) & =\sum_{l=0}^{10^{m-2}-1} G_{\alpha}^{k}\left[\frac{l}{10^{m-2}}, \frac{l}{10^{m-2}}+\frac{1}{10^{m-1}}\right) \\
& =\frac{1}{k^{-\alpha}-(k+1)^{-\alpha}} \sum_{l=0}^{10^{m-2}-1} I_{l}^{k}(m),
\end{aligned}
$$

where

$$
I_{l}^{k}(m)=\left(k+\frac{l}{10^{m-2}}\right)^{-\alpha}-\left(k+\frac{l}{10^{m-2}}+\frac{1}{10^{m-1}}\right)^{-\alpha} .
$$

If $\alpha>0$,

$$
\begin{aligned}
& \alpha\left(k+\frac{l}{10^{m-2}}+\frac{1}{10^{m-1}}\right)^{-\alpha-1} \frac{1}{10^{m-1}} \\
\leq & I_{l}^{k}(m) \leq \alpha\left(k+\frac{l}{10^{m-2}}\right)^{-\alpha-1} \frac{1}{10^{m-1}} .
\end{aligned}
$$

Since

$$
\begin{aligned}
& \left(k+\frac{l}{10^{m-2}}+\frac{1}{10^{m-1}}\right)^{-\alpha-1} \\
\geq & \left(\frac{k+\frac{l}{10^{m-2}}}{k+\frac{l}{10^{m-2}}+\frac{1}{10^{m-1}}}\right)^{\alpha+1}\left(k+\frac{l}{10^{m-2}}\right)^{-\alpha-1} \\
\geq & \left(\frac{k}{k+\frac{1}{10^{m-1}}}\right)^{\alpha+1}\left(k+\frac{l}{10^{m-2}}\right)^{-\alpha-1},
\end{aligned}
$$

we have

$$
\begin{aligned}
& \alpha\left(\frac{k}{k+\frac{1}{10^{m-1}}}\right)^{\alpha+1}\left(k+\frac{l}{10^{m-2}}\right)^{-\alpha-1} \frac{1}{10^{m-1}} \\
\leq & I_{l}^{k}(m) \leq \alpha\left(k+\frac{l}{10^{m-2}}\right)^{-\alpha-1} \frac{1}{10^{m-1}} .
\end{aligned}
$$

Therefore we see

$H_{m}^{k}(0) \sim \frac{\alpha}{k^{-\alpha}-(k+1)^{-\alpha}} \sum_{l=0}^{10^{m-2}-1}\left(k+\frac{l}{10^{m-2}}\right)^{\alpha-1} \frac{1}{10^{m-1}}$.

Using

$$
\begin{gathered}
\lim _{m \rightarrow \infty} \sum_{l=0}^{10^{m-2}-1}\left(k+\frac{l}{10^{m-2}}\right)^{-\alpha-1} \frac{1}{10^{m-2}} \\
=\int_{0}^{1}(k+x)^{-\alpha-1} d x=\frac{1}{\alpha}\left(k^{-\alpha}-(k+1)^{-\alpha}\right),
\end{gathered}
$$


we get $\lim _{m \rightarrow \infty} H_{m}^{k}(0)=10^{-1}$. Dividing $\alpha \leq 0$ into four case $\alpha=0,-1<\alpha<0$ and $\alpha \leq-1$, we can prove our assertion in the same way.

Remark 3.1. Theorem 3.1 means that the characteristic of each distribution vanishes in the distance, but to put it the other way around, the second figure exposes it exceedingly.

\section{ACKNOWLEDGMENTS}

This study started from the discussion with my colleague, Professor S. Kuriki. The author is grateful to him. He would like to thank the referee for careful reading of the manuscript and helpful comments.

Received 1 October 2013

\section{REFERENCES}

[1] Bingham, N. H., Goldie, C. M. and Teugels, J. L. (1987). Regular Variation. Cambridge. Cambridge University Press. MR0898871

[2] Fraga Alves, M. I., De Haan, L. and Neves, C. (2009). A test procedure for detecting super heavy tails. Journal of Statistical Planning and Inference 139, 213-227. MR2473999

[3] Resnick, S. I. (1987). Extreme Values, Regular Variation and Point Processes. Springer-Verlag, New York. MR0900810

Takaaki Shimura

The Institute of Statistical Mathematics

10-3 Midori-cho, Tachikawa, Tokyo 190-8562

Japan

Tel.: +81-(0)50-5533-8439

Fax: +81-(0)042-526-4335

E-mail address: shimura@ism.ac.jp 